\title{
The mortality rate of COVID-19 was high in cancer patients: a retrospective single-center study
}

\author{
Gulcin Sahingoz Erdal ${ }^{1}$ (D - Ozlem Polat ${ }^{2} \cdot$ Gokmen Umut Erdem $^{1} \cdot$ Ramazan Korkusuz $^{3} \cdot$ Fehmi Hindilerden $^{4}$.

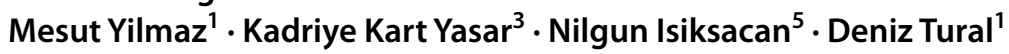

Received: 24 July 2020 / Accepted: 28 December 2020 / Published online: 24 January 2021

(c) Japan Society of Clinical Oncology 2021

\begin{abstract}
Background Coronavirus disease 2019 (COVID-19) has quickly turned into a global pandemic with close to 5 million cases and more than 320,000 deaths. Cancer patients constitute a group that is expected to be at risk and poor prognosis in COVID pandemic. We aimed to investigate how cancer patients are affected by COVID-19 infection, its clinical course and the factors affecting mortality.

Methods In our single-center retrospective study, we included cancer patients with laboratory confirmed COVID-19 in our hospital. Demographic, clinical, treatment, and laboratory data were obtained from electronic medical records. Logistic regression methods were used to investigate risk factors associated with in-hospital death.

Results In the hospital, 4489 patients were hospitalized with COVID infection and 77 were cancer patients. The mean age of cancer patients was $61.9 \pm 10.9$ and 44 of them were male $(62 \%)$. While the mortality rate in non-cancer patients was $1.51 \%$ $(n=68)$, this rate was significantly higher in cancer patients, $23.9 \%(n=17)$. The stage of the disease, receiving chemotherapy in the last 30 days also lymphopenia, elevated troponin I, D-dimer, CRP, and CT findings were associated with severe disease and mortality. Severe lung involvement $(\mathrm{OR}=22.9, p=0.01)$ and lymphopenia $(\mathrm{OR}=0.99, p=0.04)$ are the most important factors influencing survival in logistic regression.

Conclusions The disease is more severe in cancer patients and mortality is significantly higher than non-cancer patients. These data show that it may be beneficial to develop dynamic prevention, early diagnosis and treatment strategies for this vulnerable group of patients who are affected by the infection so much.
\end{abstract}

Keywords COVID-19 $\cdot$ Cancer $\cdot$ Mortality rate $\cdot$ Retrospective case study

Gulcin Sahingoz Erdal

gulcinctf@hotmail.com

1 Department of Oncology, Bakırköy dr. Sadi Konuk Training and Research Hospital, University of Health Sciences, dr. Tevfik Saglam Street No: 11, 34147 Istanbul, Turkey

2 Department of Family Medicine, Bakırköy dr. Sadi Konuk Training and Research Hospital, University of Health Sciences, Istanbul, Turkey

3 Department of Infectious Diseases and Clinical Microbiology, Bakırköy dr. Sadi Konuk Training and Research Hospital, University of Health Sciences, Istanbul, Turkey

4 Department of Hematology, Bakırköy dr. Sadi Konuk Training and Research Hospital, University of Health Sciences, Istanbul, Turkey

5 Department of Biochemistry, Bakırköy dr. Sadi Konuk Training and Research Hospital, University of Health Sciences, Istanbul, Turkey

\section{Introduction}

Since it was identified in China in December 2019, coronavirus disease 2019 (COVID-19) has quickly turned into a global pandemic with close to 5 million cases and more than 320,000 deaths [1]. After the first reported cases of Turkey on March 11, 2020, numbers continue to rise with over 1500 reported cases and over 4.000 deaths in May[2]. COVID19 causes severe acute respiratory syndrome, such as fatal coronavirus infections (severe acute respiratory syndrome coronavirus, Middle East respiratory syndrome coronavirus) that have occurred in the past 2 decades.

The first reports from China showed that the total mortality rate of COVID-19 was $1.4 \%$ [3].

The initial reports from China show that cancer patients have an important place among individuals who develop severe COVID-19 [4]. The study, which has a mortality rate 
of $28.6 \%$ in cancer patients infected with COVID-19, supports this [5].

Cancer patients constitute a group that is expected to be at risk and poor prognosis in COVID pandemic with the effect of the disease and the immune-suppressive effect of the treatment received.

However, data from China have shown that the prognosis varies greatly between groups over the age of 60 , including hypertension, diabetes, cardiovascular disease and cancer, which are identified as risk factors for serious illness and death [3].

In this retrospective study, we aimed to investigate how cancer patients are affected by COVID-19 infection, its clinical course and the factors affecting mortality.

\section{Materials and methods}

\section{Study design and participants}

Cancer patients diagnosed with COVID-19 infection in our hospital, one of the biggest pandemic centers in Istanbul, from the 15th of March to the 15th of May were included in the study. All included patients had a positive result in highefficiency sequencing or real-time reverse transcriptasepolymerase chain reaction (RT-PCR) analysis of nasal and pharyngeal swab samples. In the first days when the infection was seen in our country; since RT-PCR analysis was completed in 3-4 days, treatment was started immediately in patients whose clinical history, symptoms and chest tomography findings were compatible with COVID-19. RT-PCR analyzes were performed in accordance with the standard protocol recommended by WHO [6].

This retrospective study was approved by the Ethics of Committees of Bakirköy Sadi Konuk Training and Research Hospital, National Ethics Committees, and in accordance with the Helsinki Declaration and its later amendments or comparable ethical standards (No:2020/146).

\section{Data collection}

Clinical retrospective data were obtained from electronic medical records, including demographic features, medical history, clinical features, laboratory findings, treatments and chest computed tomography (CT) images.

\section{Definitions and laboratory methods}

The reference cell ranges accepted in our laboratory are $3700-10,100 / \mathrm{mm}^{3}$ for leukocytes, $1630-6960 / \mathrm{mm}^{3}$ for neutrophils, $1090-2990 / \mathrm{mm}^{3}$ for lymphocytes, $12.9-15.9 \mathrm{~g} / \mathrm{dl}$ for hemoglobin, $8.6-10.6 \mathrm{mg} / \mathrm{dl}$ for calcium $(\mathrm{Ca}), 35-52 \mathrm{~g} / \mathrm{l}$ for albumin, $200-400 \mathrm{mg} / \mathrm{l}$ for fibrinogen. The upper limits accepted in our lab are $0.5 \mathrm{ng} / \mathrm{ml}$ for procalcitonin, $5 \mathrm{mg} / \mathrm{l}$ for C-reactive protein (CRP), $171 \mathrm{IU} / \mathrm{L}$ for creatine kinase (CK), $6.3 \mathrm{ng} / \mathrm{ml}$ for CK-MB, $14 \mathrm{pg} / \mathrm{ml}$ for Troponin I.

Internal quality control and external quality assurance are applied to monitor the accuracy and precision of tests. All tests were performed within $2 \mathrm{~h}$ of blood collection. $\mathrm{Na}$, Albumin, LDH, CK, Troponin I, D-dimer, CRP levels were determined using Beckman Coulter AU5800 clinical chemistry analyzer (Beckman Coulter, Brea, CA, USA). A complete blood count (CBC) was analyzed with ADVIA 2120i hematology autoanalyzer (Siemens Healthcare Diagnostics, Erlangen, Germany). For coagulation assay (Fibrinogen), all analytical procedures were carried out on a random access coagulation analyzer (Beijing Succeeder Technology Inc. China) and the reagents were used according to the manufacturer's protocol.

\section{Radiological methods}

Chest CT scans were performed at the time of admission for each patient and repeated on the 14th day of treatment and if clinically required. The major CT demonstrations were described using internationally standard nomenclature defined by the Fleischner Society glossary using terms including ground-glass opacity (GGO), crazy-paving pattern, and consolidation. A semi-quantitative scoring system was used. Each of the 5 lung lobes was visually scored from 0 to 5 as: 0 , no involvement; $1,<5 \%$ involvement; $2,25 \%$ involvement; 3, 26-49\% involvement; 4, 50-74\% involvement; 5, $>75 \%$ involvement [7]. In our study, we divided it into 3 groups as mild involvement $(<25 \%$ involvement), moderate involvement (26-74\% involvement) and severe involvement ( $>75 \%$ involvement).

\section{Statistical analysis}

All statistical analysis was performed using 'Statistical Package for The Social Sciences' version 20 for Windows (SPSS, Inc, Chicago, IL, USA). For descriptive analysis, continuous variables were presented as the mean with standard deviation (SD) or as median with interquartile range (IQR), as appropriate. Categorical variables are presented as number (\%). The univariate analyses to identify variables associated with patient outcome (recover/death) during the COVID-19 infection was investigated using Chi square, Fisher exact, Student's $t$ and Mann-Whitney $U$ tests, where appropriate. $P$ value less than 0.05 was considered statistically significant. The possible factors identified with univariate analyses were further entered into the logistic regression analyses to determine independent predictors of patient outcome. Survival analysis was performed according to Kaplan-Meier Method. Overall survival (OS) was defined as the time interval 
between the diagnosis date of COVID-19 to patient's death or to last follow-up.

\section{Results}

\section{Demographic and clinical characteristics}

The 71 patients with cancer were retrospectively enrolled who were admitted to our hospitals for quarantine and treatment of COVID-19 between March 15, 2020 and May 15, 2020.

To date, 4489 of these patients have been hospitalized with the diagnosis of COVID - 19 infection, $1.58 \%(n=71)$ of inpatients had a cancer. While the mortality rate was $1.51 \%$ in non-cancer patients $(n=68)$, this rate was approximately 15 times higher in cancer patients, $23.9 \%$. The demographic and clinical characteristics of the patients were shown in Table 1.

The mean age of the patients was $61.9 \pm 10.9$ and 44 of them were male $(62 \%)$ and no significant difference between the genders in the mortality rate $(p=0.15)$.

In patients with solid organ tumors, $44.1 \%(n=26)$ of patients had cancer stage IV, and mortality was higher than the group with cancer stage I-III (55.9\%) $(p=0.03)$. Twentyseven of the patients (38\%) had received chemotherapy in the last 30 days before the pandemic, and these patients had a higher mortality rate $(p=0.04)$.

Hypertension (47.9\%) was the most common comorbid disease in cancer patients, followed by Diabetes mellitus (35.2\%). Among comorbid diseases that accompany, only hypertension was associated with mortality $(p=0.03)$.

\section{Medical history}

When patients are questioned about the medicines they use regularly; we noted that 19 patients (26.8\%) used angiotensin-converting enzyme inhibitors (ACEI) or angiotensin II receptor blocker (ARB) therapy. No significant relationship between mortality and those using ACEI or ARB $(p=0.05)$. In the last 30 days, 21 patients $(29.6 \%)$ received ibuprofen treatment, it was not associated with mortality.

All cancer patients in the study consisted of patients who were followed up regularly and continuously from the medical oncology and hematology departments of our hospital. The leading type of cancer in the patient population with COVID-19 infection was gastrointestinal cancer $(n=19$, $26.8)$, followed by genitourinary cancer $(n=12,16.9)$. Hematological malignancy was present in $16.9 \%(n=12)$ of the patients. While gastrointestinal cancer and genitourinary cancer patients each accounted for $30.8 \%$ of solid organ tumor deaths, hematological malignancy accounted for $23.5 \%$ of all deaths and no significant difference in mortality rate in hematological and non-hematological cancers. Other types of cancer and mortality rates were given in the Table 1 .

When we evaluated cancer patients according to age groups, no statistically significant difference in mortality rate.

\section{Initial symptoms and signs}

The symptoms most frequently observed at hospital admission were dyspnea (85.9\%), and fatigue, fever, cough, respectively. No significant relationship was found between patients' symptoms and mortality (Table 1).

\section{Radiological findings}

The most common patterns on chest CT were ground-glass opacity and bilateral patchy shadowing. While 67 patients had abnormal findings on chest $\mathrm{CT}$ at the time of admission (94.4\%), four patients did not have CT findings. Since these four people had a history of contact with COVID-19 infected people, the swab-PCR test was performed in the early stages and the diagnosis of infection was made before CT lung findings developed.

When we divided the patients into 3 groups of mild, moderate and severe for chest CT involvement; $18.3 \%$ of patients had severe involvement and mortality was significantly higher in these patients $(p<0.001)$ (Table 2$)$.

\section{Laboratory findings}

Laboratory findings of the patients were shown in the Table 3.

At the time of admission, $70.4 \%$ of patients had anemia, $15.5 \%$ had neutropenia and $52.1 \%$ had lymphopenia. Mortality was significantly higher in patients with lymphopenia $(p<0.001) .82 .4 \%$ of patients who died due to COVID-19 infection had lymphopenia at the time of admission to the hospital, and the mortality rate in patients with lymphopenia was $38.9 \%$.

$39.4 \%$ of patients had hypocalcemia, $59.2 \%$ had hypoalbuminemia, $59.2 \%$ had elevated fibrinogen, $43.7 \%$ had elevated D-dimer and $29 \%$ had elevated troponin level. Elevated D-dimer, procalcitonin, CRP and elevated Troponin levels showed a significant relationship with mortality at the first hospital admission.

\section{Treatment and clinical course}

The treatments applied to patients and their relationship with mortality were given in the Table 2 and no relationship between the treatments and mortality.

Oxygen therapy was applied to a total of 64 patients (90.1\%). During the follow-up in the hospital, 17 (23.9\%) 
Table 1 Demographic and base line clinical characteristics of COVID-19-infected cancer patients

\begin{tabular}{|c|c|c|c|c|}
\hline Characteristics & Patients $n=71$ & Recovery $n=54$ & Death $n=17$ & $p$ \\
\hline Mean age (years) & $61.9 \pm 10.9$ & $61.2 \pm 11.2$ & $63.6 \pm 10.1$ & 0.97 \\
\hline \multicolumn{5}{|l|}{ Age } \\
\hline $30-49$ & $9(12.7)$ & $8(14.8)$ & $1(5.9)$ & \\
\hline $50-69$ & $42(59.2)$ & $32(59.3)$ & $10(58.8)$ & 0.54 \\
\hline$\geq 70$ & $20(28.2)$ & $14(25.9)$ & $6(35.3)$ & \\
\hline \multicolumn{5}{|l|}{ Gender } \\
\hline Male & $44(62)$ & $31(57.4)$ & $13(76.5)$ & 0.15 \\
\hline Female & $27(38)$ & $23(42.6)$ & $4(27.0)$ & \\
\hline \multicolumn{5}{|l|}{ Comorbidities } \\
\hline Hypertension & $34(47.9)$ & $22(40.7)$ & $12(70.6)$ & 0.03 \\
\hline Diabetes mellitus & $25(35.2)$ & $18(33.3)$ & $7(41.2)$ & 0.55 \\
\hline COPD & $20(28.2)$ & $14(25.9)$ & $6(35.3)$ & 0.54 \\
\hline Renal insufficiency & $7(9.9)$ & $5(9.3)$ & $2(11.8)$ & 0.67 \\
\hline Cerebrovascular disease & $6(8.5)$ & $3(5.6)$ & $3(17.6)$ & 0.14 \\
\hline Others & $11(15.5)$ & $8(14.8)$ & $3(17.6)$ & 0.71 \\
\hline $\mathrm{ACE}$ inhibitor or $\mathrm{ARB}$ & $19(26.8)$ & $11(20.4)$ & $8(47.1)$ & 0.05 \\
\hline İbuprofen & $21(29.6)$ & $17(31.5)$ & $4(23.5)$ & 0.53 \\
\hline \multicolumn{5}{|l|}{ Site of cancer } \\
\hline Hematologic cancer* & $12(16.9)$ & $8(14.8)$ & $4(23.5)$ & 0.40 \\
\hline Non-hematologic cancer & $59(83.1)$ & $46(85.2)$ & $13(76.5)$ & \\
\hline \multicolumn{5}{|c|}{ Tumor stage (Non-hematologic cancer) } \\
\hline Stage I-III & $33(55.9)$ & $29(63.0)$ & $4(30.8)$ & $\mathbf{0 . 0 3}$ \\
\hline Stage IV & $26(44.1)$ & $17(37.0)$ & $9(69.2)$ & \\
\hline \multicolumn{5}{|l|}{ Tumor diagnosis } \\
\hline Gastrointestinal cancer & $19(26.8)$ & $15(27.8)$ & $4(23.5)$ & \\
\hline Lung cancer & $7(9.9)$ & $6(11.1)$ & $1(5.9)$ & \\
\hline Genitourinary cancer & $12(16.9)$ & $8(14.8)$ & $4(23.5)$ & 0.67 \\
\hline Breast cancer & $11(15.5)$ & $10(18.5)$ & $1(5.9)$ & \\
\hline Others $^{\#}$ & $10(14.1)$ & $7(13.0)$ & $3(17.6)$ & \\
\hline Hematologic cancer & $12(16.9)$ & $8(14.8)$ & $4(23.5)$ & \\
\hline \multicolumn{5}{|c|}{ Anti-cancer therapy within 1 month } \\
\hline Yes & $27(38.0)$ & $17(31.5)$ & $10(58.8)$ & 0.04 \\
\hline No & $44(62.0)$ & $37(68.5)$ & $7(41.2)$ & \\
\hline \multicolumn{5}{|l|}{ Sympthoms } \\
\hline Cough & $49(69.0)$ & $36(66.7)$ & $13(76.5)$ & 0.44 \\
\hline Fever (above 37.8) & $50(70.4)$ & $37(68.5)$ & $13(76.5)$ & 0.53 \\
\hline Lassitude and fatigue & $58(81.7)$ & $43(79.6)$ & $15(88.2)$ & 0.72 \\
\hline Shortness of breath & $61(85.9)$ & $46(85.2)$ & $15(88.2)$ & 1.0 \\
\hline Headache & $15(21.1)$ & $9(16.7)$ & $6(35.3)$ & 0.16 \\
\hline Myalgia-Arthralgia & $18(25.4)$ & $11(20.4)$ & $7(41.2)$ & 0.11 \\
\hline Myocarditis & $1(1.4)$ & $0(0.0)$ & $1(5.9)$ & 0.23 \\
\hline Loss of taste & $1(1.4)$ & $1(1.9)$ & $0(0.0)$ & 1.0 \\
\hline Others & $10(14.1)$ & $6(11.1)$ & $4(23.5)$ & 0.23 \\
\hline
\end{tabular}

ACE Angiotensin-converting enzyme; ARB angiotensin II receptor blocker; COPD chronic obstructive pulmonary disease

*Chronic lymphocytic leukemia (4), acute myeloid leukemia (3), lymphoma (3), chronic myeloid leukemia (1), myelodysplastic syndrome (1)

${ }^{\#}$ Papillary thyroid cancer (3), head and neck cancer (2), malignant melanoma (2), brain tumor (2), adrenocortical carcinoma (1) 
Table 2 Treatment and clinical outcome of the patients

\begin{tabular}{lccrr}
\hline Characteristics & Patients $n=71$ & Recovery $n=54$ & Death $n=17$ & $p$ \\
\hline Treatment regimen & & & & \\
Antibiotic use & $67(94.4)$ & $52(96.3)$ & $15888.2)$ & 0.24 \\
Hydroxychloroquine & $71(100)$ & $54(100)$ & $17(100)$ & \\
Oseltamivir & $52(73.2)$ & $38(70.4)$ & $14(82.4)$ & 0.53 \\
Favipavir & $27(38.0)$ & $18(33.3)$ & $9(52.9)$ & 0.14 \\
Lopinavir & $3(4.2)$ & $1(1.9)$ & $2(11.8)$ & 0.14 \\
Tocilizumab & $6(8.5)$ & $3(5.6)$ & $3(17.6)$ & 0.14 \\
Vitamin C & $14(19.7)$ & $12(22.2)$ & $2(11.8)$ & 0.49 \\
Plasma theraphy & $1(1.4)$ & $1(1.9)$ & $0(0.0)$ & 1.00 \\
Admission to ICU & $18(25.4)$ & $2(3.7)$ & $16(94.1)$ & $<\mathbf{0 . 0 0 1}$ \\
Mechanical ventilation (\%) & $17(23.9)$ & $1(1.9)$ & $16(94.1)$ & $<\mathbf{0 . 0 0 1}$ \\
Chest CT findings & & & & \\
No & $4(5.6)$ & & & \\
Yes & $67(94.4)$ & & & \\
$\quad$ Mild & $36(50.7)$ & $33(66.0)$ & $3(17.6)$ & \\
Moderete & $18(25.4)$ & $13(26.0)$ & $5(29.4)$ & $<\mathbf{0 . 0 0 1}$ \\
Severe & $13(18.3)$ & $4(8.0)$ & $9(52.9)$ & \\
Median incubation period (days) & $6.0(1-28)$ & $26.0(26.0)$ & $6.0(1-28)$ & 0.14 \\
Median lenght of hospital stay (days) & $10.0(1-39)$ & $9.5(1-39)$ & $11.0(1-30)$ & 0.58 \\
Median length of ICU stay (days) & $7.5(1-32)$ & $22.0(12-32)$ & $6.0(1-28)$ & $\mathbf{0 . 0 5}$ \\
\hline
\end{tabular}

CT Computed tomography; ICU intensive care unit

Table 3 Laboratory findings on the day of admission

\begin{tabular}{|c|c|c|c|c|}
\hline Patient characteristics & Patients $n=71$ & Recovery $n=54$ & Death $n=17$ & $p$ \\
\hline White-cell count (per $\mathrm{mm}^{3}$ ) & $6440(580-32,130)$ & $6340(580-24,880)$ & $7150(1290-32,130)$ & 0.71 \\
\hline Neutrophil count (per mm³) & $3980(100-30,150)$ & $3925(100-22,790)$ & $6080(720-30,150)$ & 0.87 \\
\hline Lymphocyte count (per $\mathrm{mm}^{3}$ ) & $990(30-4220)$ & $1330(230-4220)$ & $530(30-1270)$ & $<0.001$ \\
\hline Hemoglobin $(g / d l)$ & $10.7(4.5-16.0)$ & $11.1(4.5-16.0)$ & $10.6(4.5-15.7)$ & 0.33 \\
\hline Platelet count (per $\mathrm{mm}^{3}$ ) & $199.000(19.000-582.000)$ & $208.000(19.000-582.000)$ & $165.000(30.000-437.000)$ & 0.12 \\
\hline Serum calcium (mg/dl) & $8.7(6.8-10.4)$ & $8.8(6.8-10.4)$ & $8.6(6.9-10.2)$ & 0.29 \\
\hline Albumin $(\mathrm{g} / \mathrm{L})$ & $32(16-48)$ & $33(17-48)$ & $29(16-46)$ & 0.15 \\
\hline Fibrinogen (mg/dl) & $442(186-905)$ & $437(186-818)$ & $501(211-905)$ & 0.26 \\
\hline D-Dimer (ng/ml) & $0.46(0.03-7.3)$ & $0,34(0.03-7.3)$ & $2.07(0.26-6.2)$ & $<0.001$ \\
\hline Procalcitonin (ng/ml) & $0.19(0.01-20.9)$ & $0.13(0.01-3.51)$ & $0.99(0.04-20.9)$ & $<0.001$ \\
\hline $\mathrm{CRP}(\mathrm{mg} / \mathrm{dl})$ & $76(1-294)$ & $56(1-294)$ & $157(15-277)$ & $<0.001$ \\
\hline Creatine kinase (IU/L) & $66(5.0-2062)$ & $68(5.0-2062)$ & $65(21-458)$ & 0.95 \\
\hline CK-MB (ng/ml) & $1.8(0.6-79.4)$ & $1.8(0.6-79.4)$ & $2.4(1.1-25.0)$ & 0.64 \\
\hline Troponin (pg/ml) & $7.0(2-1417)$ & $5.5(2-1417)$ & $24(5-1276)$ & $<0.001$ \\
\hline
\end{tabular}

patients underwent invasive mechanical ventilation, endotracheal intubation, and were followed up in the intensive care unit (ICU) due to progressive hypoxia. The median period of invasive mechanical ventilation in ICU was 6 days (Table 2).

Fifty-four of the patients recovered $(76 \%)$ and were discharged, $94.1 \%$ of patients followed in ICU could not be saved $(n=16)$.

\section{Risk factors for predicting death}

Logistic regression analysis results showed the most important factors influencing survival were severe lung involvement $(\mathrm{OR}=22.9, p=0.01)$ and lymphopenia $(\mathrm{OR}=0.99$, $p=0.04$ ) (Table 4). It has been shown the survival proportions according to lymphopenia and chest CT findings in Figs. 1, 2, respectively. 
Table 4 Logistic regression model for identifying risk factors for predicting death

\begin{tabular}{lccc}
\hline Variables & OR & $95 \%$ CI & $p$ \\
\hline Hypertension & 1.38 & $0.11-17.2$ & 0.80 \\
Tumor stage & 0.66 & $0.46-9.60$ & 0.76 \\
$\begin{array}{l}\text { Anti-cancer therapy } \\
\quad \text { within one month }\end{array}$ & 0.46 & $0.35-6.18$ & 0.56 \\
Chest CT findings & $\mathbf{2 2 . 9}$ & $2.00-261.82$ & $\mathbf{0 . 0 1}$ \\
$\quad$ (mild, moderete vs. & & & \\
$\quad$ severe) & & & \\
Lymphocyte & $\mathbf{0 . 9 9}$ & $0.995-0.999$ & $\mathbf{0 . 0 4}$ \\
D-Dimer & 1.96 & $0.93-4.13$ & 0.07 \\
Procalcitonin & 1.03 & $0.59-1.79$ & 0.91 \\
CRP & 1.00 & $0.99-1.01$ & 0.57 \\
Troponin & 1.02 & $0.99-1.05$ & 0.15 \\
\hline
\end{tabular}

CI Confidence interval; $O R$ odds ratio

\section{Discussion}

In our single-center study, we evaluated the status of cancer patients infected with COVID-19 at Bakirkoy Dr Sadi Konuk Training and Research Hospital, one of the largest pandemic hospitals in Istanbul. The mortality rate is $1.51 \%$ in COVID-19 patient population in our hospital. As expected, the mortality in cancer patients is dramatically higher when compared to the non-cancer population and this rate is $23.9 \%$. It is similar to the analysis of cancer patients in Wuhan, China. In the current study, the mortality rate was slightly higher than ours, with $28.6 \%$ [5]. In another study including 52 COVID-19 infected cancer patients in China, mortality rate was found to be $21.2 \%$, similar to other studies [8]. This mortality rate is more than ten times higher than reported in all COVID-19 patients in China, just like ours [9]. Initial case series analyses from China and Italy suggested that patients with malignancy would have more severe COVID-19 infection and higher mortality [4, 10-12] as in previous pandemics [13].

In an analysis in New York City, from March 1 to April 6,2020 , cancer patients were found to be significantly more frequently intubated without relative adjustments for age groups, but the mortality rate was not significantly different. When patients were grouped by age, it was seen that the risk of intubation was significantly higher in cancer patients aged 66-80 years and no significant difference in other age groups [14]. In our patient population, there was no significant difference between age groups in terms of intubation and mortality. The high average age of our patients and the low number of young patients may contribute to this situation.

In the cohort, which included 218 COVID-19 cancer patients in New York City, a higher mortality rate was observed in hematological malignancies than solid tumors [14], but there was no such difference in our patient group. This may be due to the low number of hematological malignancy patients $(n=12)$ in our study and most of these patients are in remission.
Fig. 1 The survival proportions according to chest CT findings

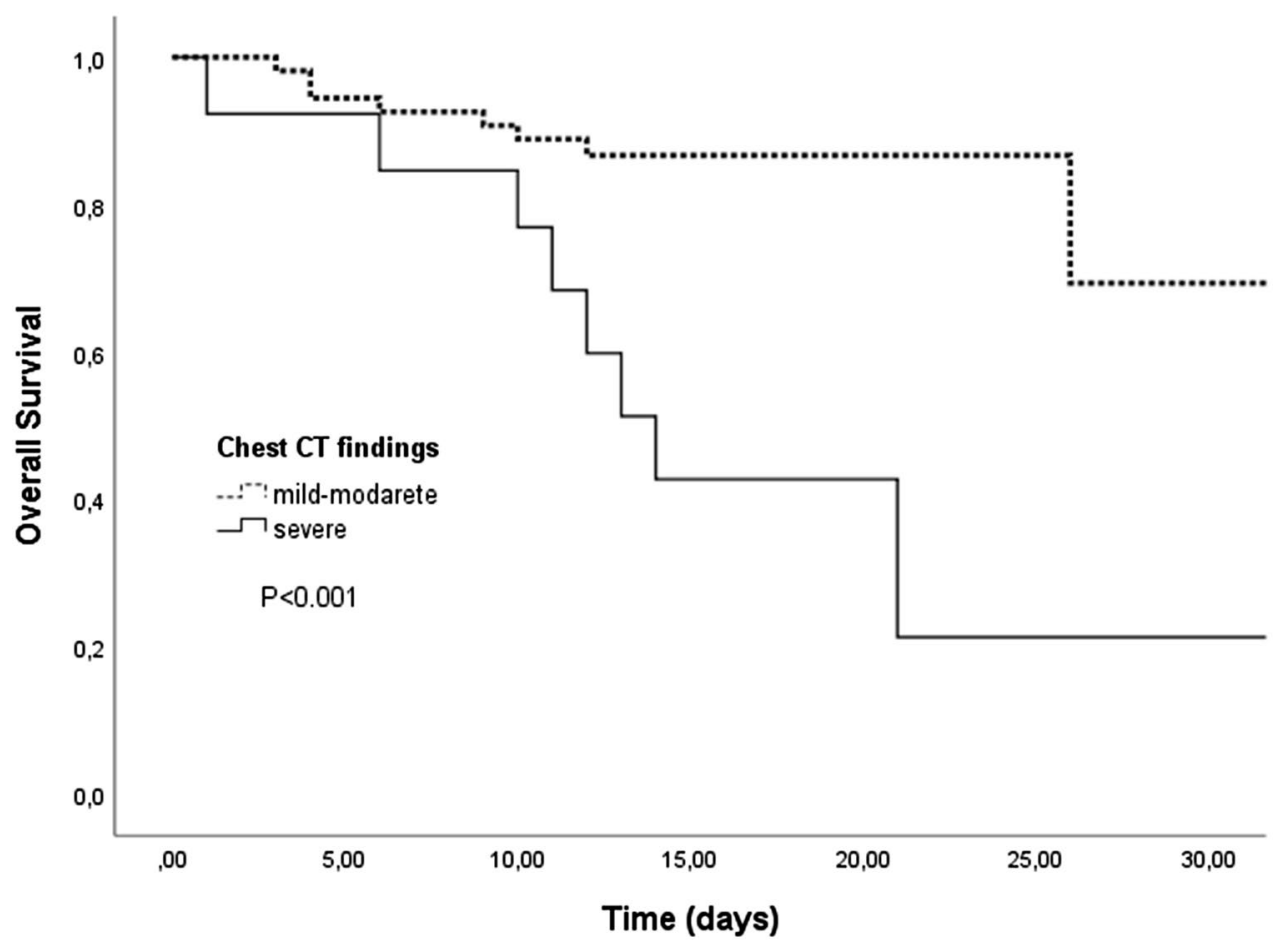


Fig. 2 The survival proportions according to pretreatment lymphocyte counts

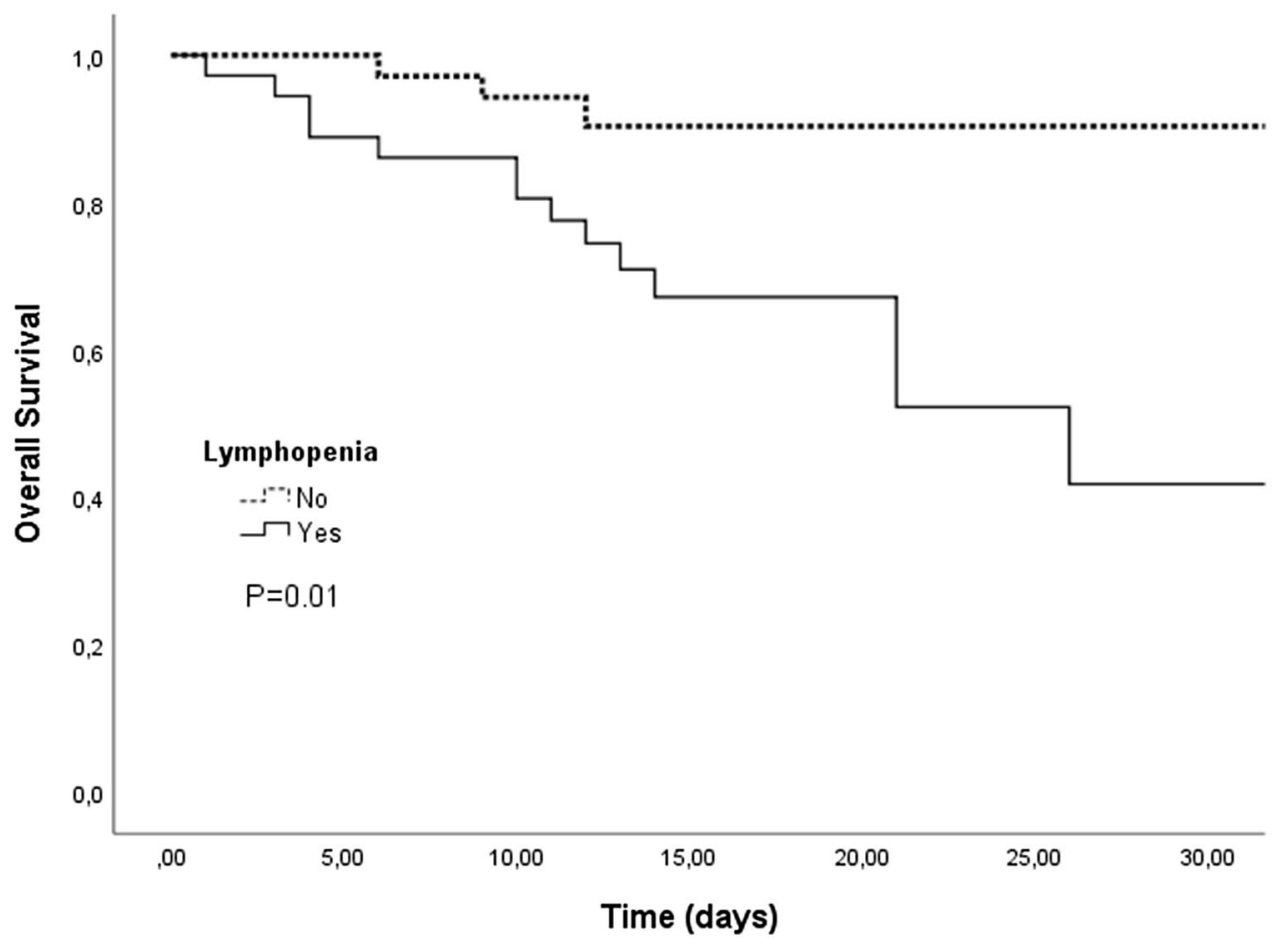

In addition, in the cohort study involving 28 cancer patients in China [5] and in the another study of multicenter involving 105 cancer patients in China [15]; the most common type of cancer with the highest mortality rate was lung cancer but contrary to expectations, this was gastrointestinal malignancy in our patient population.

The data in our hospital are compatible with the multicenter cohort study conducted in China, and as in this cohort, patients with metastatic cancer (stage IV) had a higher risk of death. And also anti-cancer treatment in the last 30 days was associated with an increased risk of death (an anti-cancer treatment in the last 40 days, in the cohort in China was evaluated) [15]. In addition, Zhang et al. determined that the use of anti-cancer treatments in the past 14 days (including chemotherapy, immunotherapy and radiation) is an independent predictor of death or other serious events with a greater than 4 hazard rate [5]. In the light of these data, postponing adjuvant chemotherapy or reducing the dose may be considered in pandemic period, especially in high-risk patients.

Chest CT involvement of our patients correlates with the severity of the disease and mortality is higher in those with severe involvement in CT also there is a similar relationship in the studies of Zhou et al. and Zhang et al. [5, 16]. Because almost all of our patients present with pneumonia, chest CT involvement is very important in predicting the severity of the disease and overall survival. When patients first apply to the hospital, chest CT can help clinicians to judge the severity of the patient and evaluate the prognosis.
In the study of Li et al. [17], the relationship between CT findings and disease severity of 83 COVID infected patients was investigated, and it was observed that the severity of CT involvement was compatible with the severity of the disease.

Our results showed that the laboratory tests of cancer patients who died had the following characteristics: low lymphocytes, increased CRP, PCT, D-dimer and Troponin, these finds were similar to previous studies [5, 8, 18]. Since the elevated D-dimer level is closely related to thromboembolic events, it is inevitable to expect additional complications in these patients [19]. It is known that elevated D-dimer levels were associated with poor overall survival and increased mortality risk in cancer patients [20]. The inflammatory response plays a critical role in COVID-19, and inflammatory cytokine storm increases the severity. The serum levels CRP and increased procalcitonin values can effectively assess disease severity and predict outcome in patients with COVID-19 [21, 22]. High troponin value may be beneficial for attention to cardiovascular effects in COVID-19 infection [23]. Lymphocytes play a decisive role in maintaining immune homeostasis and the inflammatory response in the body. Studies have shown that lymphopenia is an effective and reliable indicator of the severity and hospitalization in COVID-19 patients [3, 5, 8, 24]. On the contrary, there is also a study in which lymphopenia is not associated with mortality [14]. In our study, the lymphocyte level among laboratory findings was associated with mortality and overall survival in logistic regression, and mortality increases as the lymphocyte level decreases $(\mathrm{OR}=0.99, p=0.04)$. 
It is obvious that cancer patients are at a much higher risk in this COVID-19 outbreak than those non-cancer. The suggestions made on this subject are to consider postponing adjuvant chemotherapy in endemic regions or elective surgery for stable cancer. More careful surveillance or treatment should be considered in cancer patients when patients are infected with COVID-19, especially in elderly patients or those with other comorbidities. And the personal protection measures must be taken more carefully in cancer patients [4]. In our hospital, adjuvant chemotherapies were postponed in appropriate patients during the epidemic period, the patient population in the chemotherapy unit was diluted, paying attention to social distance, cancer patients infected with COVID-19, independent of disease severity, were hospitalized and treated. During this period, the cancer treatment of 27 patients (38\%) hospitalized for COVID-19 infection was discontinued or postponed.

There were several limitations to our study. Our study is single-centered, and because the number of patients is low, cancer distribution does not coincide with the distribution in our country and this limited population may not cover the general population. Our study only included patients with moderate or severe infection admitted to the hospital so the frequency of COVID-19 infection in cancer patients who do not have a serious infection and do not apply in the hospital is unknown. Despite all the limitations, it is the largest series of COVID-19-infected cancer patient in a single center we know so far.

\section{Conclusions}

COVID-19 disease is more severe in cancer patients and mortality is significantly higher than non-cancer patients. These data show that it may be beneficial to develop dynamic prevention, early diagnosis and treatment strategies for this vulnerable group of patients who are affected by the infection so much.

Acknowledgment The authors have declared no conflicts of interest.

Author contributions GSE, OP, GUE, RK, FH, MY, KKY, NI, and DT contributed to the design and implementation of the research, to the analysis of the results and to the writing of the manuscript.

Funding There is no funding.

\section{Compliance with ethical standards}

Conflict of interest The authors have declared no conflicts of interest.

Ethical approval This retrospective study was approved by the Ethics of Committees of Bakirköy Sadi Konuk Training and Research Hospital, National Ethics Committees, and in accordance with the Helsinki
Declaration and its later amendments or comparable ethical standards (No:2020/146).

Consent for publication All authors gave their consent for publication of this scientific article.

\section{References}

1. World Health Organization (2020) World health organization. Coronavirus disease Coronavirus disease (COVID-2019) situation reports. https://www.who.int/emergencies/diseases/novel -coronavirus-2019/situation-reports/

2. Coronavirus Updates. In. 2020. https://www.worldometers.info/ coronavirus/

3. Guan W-j, Ni Z-y, Hu Y et al (2019) Clinical characteristics of coronavirus disease, in China. N Engl J Med 382:1708-1720

4. Liang W, Guan W, Chen R et al (2020) Cancer patients in SARS-CoV-2 infection: a nationwide analysis in China. Lancet Oncol 21:335-337

5. Zhang L, Zhu F, Xie L et al (2020) Clinical characteristics of COVID-19-infected cancer patients: A retrospective case study in three hospitals within Wuhan China. Ann Oncol 2:19

6. Organization WH (2020) Laboratory testing for coronavirus disease 2019 (COVID-19) in suspected human cases: interim guidance. In. World Health Organization, pp 1-9

7. Pan F, Ye T, Sun P et al (2019) Time course of lung changes on chest CT during recovery from, novel coronavirus (COVID-19) pneumonia. Radiology 2:200370

8. Yang F, Shi S, Zhu J et al (2020) Clinical characteristics and outcomes of cancer patients with COVID-19. J Med Virol 92(10):2067-2073

9. Wu Z, McGoogan JM (2020) Characteristics of and important lessons from the coronavirus disease 2019 (COVID-19) outbreak in China: summary of a report of 72314 cases from the Chinese center for disease control and prevention. JAMA 323:1239-1242

10. Xia Y, Jin R, Zhao J et al (2020) Risk of COVID-19 for patients with cancer. Lancet Oncol 21:e180

11. Wang H, Zhang L (2020) Risk of COVID-19 for patients with cancer. Lancet Oncol 21:e181

12. Onder G, Rezza G, Brusaferro S (2020) Case-fatality rate and characteristics of patients dying in relation to COVID-19 in Italy. JAMA 23(18):1775-1776

13. Dignani MC, Costantini P, Salgueira C et al (2014) Pandemic 2009 Influenza A (H1N1) virus infection in cancer and hematopoietic stem cell transplant recipients; a multicenter observational study. Research 14:3

14. Mehta V, Goel S, Kabarriti R et al (2020) Case fatality rate of cancer patients with COVID-19 in a New York hospital system. Cancer Discov 10(7):935-941

15. Dai M, Liu D, Liu M et al (2020) Patients with cancer appear more vulnerable to SARS-COV-2: a multicenter study during the COVID-19 outbreak. Cancer Dis 10(6):783-791

16. Zhou F, Yu T, Du R et al (2020) Clinical course and risk factors for mortality of adult inpatients with COVID-19 in Wuhan, China: a retrospective cohort study. The Lancet 395(10229):1054-1062

17. Li K, Wu J, Wu F et al (2020) The clinical and chest CT features associated with severe and critical COVID-19 pneumonia. Invest Radiol 55:327-331

18. Ma J, Yin J, Qian Y, Wu Y (2020) Clinical characteristics and prognosis in cancer patients with COVID-19: A single center's retrospective study. J Infect 81(2):318-356 
19. Gao Y, Li T, Han M et al (2020) Diagnostic utility of clinical laboratory data determinations for patients with the severe COVID-19. J Med Virol 92(7):791-796

20. Ay C, Dunkler D, Pirker R et al (2012) High D-dimer levels are associated with poor prognosis in cancer patients. Haematologica 97:1158-1164

21. Liu F, Li L, Xu M et al (2020) Prognostic value of interleukin-6, C-reactive protein, and procalcitonin in patients with COVID-19. J Clin Virol 2:104370

22. Emami A, Javanmardi F, Pirbonyeh N, Akbari A (2020) Prevalence of underlying diseases in hospitalized patients with COVID19: a systematic review and meta-analysis. Arch Acad Emerg Med $8: 4$
23. Tersalvi G, Vicenzi M, Calabretta D et al (2020) Elevated troponin in patients with Coronavirus Disease 2019 (COVID-19): possible mechanisms. J Cardiac Failure 26(6):470-475

24. Tan L, Wang Q, Zhang D et al (2020) Lymphopenia predicts disease severity of COVID-19: a descriptive and predictive study. Signal Trans Targ Thera 5:1-3

Publisher's Note Springer Nature remains neutral with regard to jurisdictional claims in published maps and institutional affiliations. 